\title{
Cardiomyocytes acting like adults
}

\section{By Tracey Baas, Senior Editor}

A collaboration between Sanford-Burnham Medical Research Institute and The Johns Hopkins University School of Medicine cardiology teams has developed a model for arrhythmogenic right ventricular dysplasia/cardiomyopathy that for the first time is able to replicate the phenotype of the disease. ${ }^{1}$ The key innovation was getting the induced pluripotent stem cell-based model to behave more like adult heart cells than fetal heart cells.

The team expects that the model could be used to identify diseasemodifying therapeutics and thinks researchers working with induced pluripotent stem (iPS) cell-based models of other disease should consider maturing the final cell type when needed.

Arrhythmogenic right ventricular dysplasia/cardiomyopathy (ARVD/C) occurs in about 1 in 5,000 people, with onset typically occurring at 20-40 years of age. The disease is characterized by progressive right ventricular myocardium degeneration and apoptosis, ventricular arrhythmias, fibrous-fatty replacement of cardiac muscle within the right ventricular wall and increased risk of sudden death. ${ }^{2}$

Understanding the pathogenesis

"In cardiac drug development, the vast majority of preclinical testing is conducted with animal or immortalized cell lines that do not resemble the human heart."

-Joseph Wu,

Stanford University School of Medicine of ARVD/C has been challenging. For example, it is hard to obtain tissues from patients with ARVD/C at various stages of the disease because cardiac biopsies are dangerous and patients are commonly diagnosed at late stages of disease or even postmortem.

"In cardiac drug development, the vast majority of preclinical testing is conducted with animal or immortalized cell lines that do not resemble the human heart. In most cases, the first time a pharmacological compound interacts directly with a human cardiomyocyte is in a Phase I trial," said Joseph $\mathrm{Wu}$, co-director of the Stanford Cardiovascular Institute and associate professor of medicine and radiology at the Stanford University School of Medicine.

To remedy this, the Sanford-Burnham group, with help from the Johns Hopkins Center for Inherited Heart Diseases, opted to produce iPS cell-derived cardiomyocytes. The team generated iPS cells from the fibroblasts of patients with ARVD/C who had plakophilin 2 (PKP2) mutations and then differentiated the cells into cardiomyocytes using previously published protocols. ${ }^{3,4}$

Mutations in junction proteins, called desmosomes, are associated with ARVD/C. In addition to PKP2, ARVD/C-associated mutations also have been identified in subunits of desmosomes on cardiac cells that help glue the cells together, including junction plakoglobin (JUP), desmoplakin (DSP), desmoglein 2 (DSG2) and desmocollin 2 (DSC2). ${ }^{2}$

Curiously, the mutant PKP2 cardiomyocytes did not reproduce pathological ARVD/C. The group attributed the lack of a pathologic phenotype to an absence of exaggerated lipogenesis and apoptosis, which are found in adult-like but not fetal-like cardiomyocytes.

Indeed, the mutant PKP2 cardiomyocytes had qualities that were similar to those of fetal-like cells, including using mostly glycolysis for energy production rather than fatty acid oxidation.,

To induce adult-like metabolism and accelerate pathogenesis in the mutant $P K P 2$ iPS cell-derived cardiomyocytes, the team created a lipogenic cocktail. The cocktail resulted in mutant $P K P 2$ iPS cell-derived cardiomyocytes with adult-like metabolism, exaggerated lipogenesis and pronounced apoptosis.

The cardiomyocytes with pathologies also showed impaired calcium relaxation and electrophysiological properties in normal media, which may contribute to the cellular pathology and arrhythmia in patients with ARVD/C.

The group's final step was making the iPS cell-derived cardiomyocytes specifically resemble cells found in the right cardiac ventricle-the predominant pathological location of ARVD/C. To do so, the group added 6-bromoindirubin- $3^{\prime}$-oxime to the cocktail. This reagent increases levels of islet 1-positive cardiac progenitor cells and subsequent cardiomyocytes, which form the right ventricle.?

Mutant cardiomyocytes derived from enriched islet 1-positive cardiac progenitor cells showed significantly greater lipogenesis and apoptosis than cells derived from unenriched progenitor cells.

Results were published in Nature.

"The approach that the team employs is especially interesting because the authors induce maturation in the iPS cell-derived cardiomyocytes in order to observe the disease phenotype," said Wu. "This approach may be employed to model both cardiac and noncardiac diseases in the future given that many of the embryonic stem cell and iPS cell derivatives made today in laboratories resemble fetal cells more closely than adult counterparts."

"That they found a metabolic switch to transition from fetal-like cardiac cells to adult-like cardiac cells is a major breakthrough and will provide a very valuable model," said Roberto Iacone, laboratory head of the stem cell, cardiovascular and metabolism group at Roche. "It is encouraging to see this advance. We are using a similar approach to develop our own stem cell-based models and are genetically and pharmacologically altering the insulin pathway in order to provide a more translational cardiomyocyte platform to study the signaling pathways responsible for type 2 diabetes [-related] cardiovascular complications."

\section{Taken to heart}

Huei-Sheng Vincent Chen, associate professor of neuroscience, aging 
and stem cell research at Sanford-Burnham and principal investigator of the new study, thinks maturation will probably be needed for many iPS cell-based systems-especially those used to represent adult-onset diseases.

"Although a number of iPS cell-derived neurons in a dish show some disease phenotypes-such as those for Alzheimer's, Parkinson's and Huntington's-we don't know if this is as good as it gets because no detailed genetic, metabolic or cellular maps are established to guide the maturation induction from human fetal neurons to adult neurons, especially regarding their metabolic states," Chen noted.

"A number of groups have provided iPS cell-based models for Alzheimer's and long QT syndrome, but it would be interesting to see if incorporating a maturation step would provide a more pathogenic phenotype with more similarities to the human disease," added Sheng Ding, a senior investigator at the Gladstone Institute of Cardiovascular Disease, a professor of pharmaceutical chemistry at the University of California, San Francisco and a cofounder of stem cell company Fate Therapeutics Inc.

Currently, Chen's team has only checked one box in the list of ways to make iPS cell-derived cardiomyocytes mature.

"Right now, we have only dug into one aspect of maturationmetabolism. In fact, adequate human disease modeling with iPS cell derivatives might require many other aspects of maturation induction, which could include transverse tubule networks that provide structure, gap junction distribution, cell size and morphology," said Chen. "I would be extremely happy if other teams could use what we learned from cardiomyocytes to develop other types of mature cells derived from iPS cells. We still have quite a distance to go."

Chris Parker, VP and chief commercial officer at stem cell company Cellular Dynamics International Inc., cautioned that it is possible to go overboard in the maturation of cardiomyocytes. "We've been using our own media cocktails since 2009 that switch newly differentiated cardiomyocytes into a functional cardiomyocyte phenotype," he said. "Our functional cardiomyocyte phenotype resembles many aspects of cells found in an adult human heart, but they are adult-like and not adult. You may not want a fully adult cardiomyocyte because they do not spontaneously beat in culture, and this spontaneous beating is an important characteristic needed to screen for efficacy and toxicity of potential therapeutics."

He also said that a maturation cocktail that is useful for cardiomyocytes "would not be useful for other cell types. For each of our cell typescardiomyocytes, endothelial cells, hepatocytes and neurons-we have specific media to provide cells to customers with appropriate functional phenotypes."

Chen's team has filed for patents covering its work. The licensing status of the IP is undisclosed. Chen said he would like to find a partner to distribute the group's model iPS cells so that they can be used to develop ARVD/C-modifying therapeutics.

Baas, T. SciBX 6(8); doi:10.1038/scibx.2013.183

Published online Feb. 28, 2013

\section{REFERENCES}

1. Kim, C. et al. Nature; published online Jan. 27, 2013; doi:10.1038/nature11799

Contact: Huei-Sheng Vincent Chen, Sanford-Burnham Medical Research Institute, La Jolla, Calif. e-mail: hsv_chen@burnham.org

2. Basso, C. et al. Nat. Rev. Cardiol. 9, 223-233 (2012)

3. Takahashi, K. et al. Cell 131, 861-872 (2007)

4. Okita, K. et al. Nat. Methods 8, 409-412 (2011)

5. Onay-Besikci, A. Mol. Cell. Biochem. 287, 1-11 (2006)

6. Lopaschuk, G.D. et al. Physiol. Rev. 90, 207-258 (2010)

7. Cai, C.-L. et al. Dev. Cell 5, 877-889 (2003)

\section{COMPANIES AND INSTITUTIONS MENTIONED}

Cellular Dynamics International Inc., Madison, Wis. Fate Therapeutics Inc., San Diego, Calif. Gladstone Institute of Cardiovascular Disease, San Francisco, Calif.

The Johns Hopkins University School of Medicine, Baltimore, Md.

Roche (SIX:ROG; OTCQX:RHHBY), Basel, Switzerland Sanford-Burnham Medical Research Institute, La Jolla, Calif. Stanford University School of Medicine, Stanford, Calif. University of California, San Francisco, Calif. 